\title{
CALCUL EXPLICITE DE CERTAINES CELLULES DE KAZHDAN-LUSZTIG POUR LE TYPE $A_{n-1}$
}

\author{
par \\ Nicolas Jacon \\ Résumé. - The aim of this paper is to give an explicit characterization of the Kazhdan-Lusztig \\ cells which contains the elements with maximal length in parabolic subgroups of $\mathfrak{S}_{n}$.
}

Table des matières

Introduction.............................................. 1

1. Cellules de Kazhdan-Lusztig pour le groupe symétrique, correspondance de

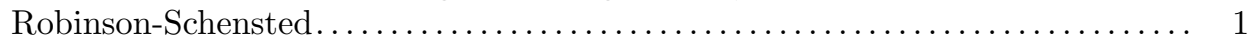

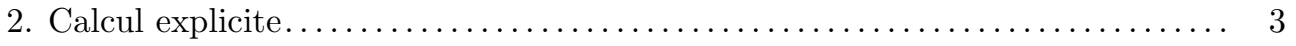

Références................................................ 6

\section{Introduction}

Soit $W$ un groupe de Coxeter. Dans $[\mathbf{K L}]$, Kazhdan et Lusztig ont donné un moyen de partitionner $W$ en "cellules à gauche". Ces cellules jouent un rôle fondamental par exemple dans la théorie des représentations des algèbres de Hecke et des groupes de Lie (voir par exemple [Lu2]). Dans le cas du groupe symétrique, groupe qui nous intéresse ici (c'est à dire lorsque $W=A_{n-1}$ avec $n \in \mathbb{N}$ ), la correspondance de Robinson-Schensted fournit un algorithme relativement simple pour la détermination de ces cellules. Cependant, il pourrait être intéressant de déterminer explicitement l'ensemble des éléments appartenant à une cellule donnée.

Le but de cette note est de résoudre ce problème pour une certaine classe de cellules : les cellules contenant un élément de longueur maximal dans un sous-groupe parabolique de la forme $\mathfrak{S}_{\lambda}$ où $\lambda$ est une partition de $n$. Dans la première partie, nous rappelons la définition de ces cellules (pour le type $A_{n-1}$ ) et de la correspondance de Robinson-Schensted. Puis, nous déterminons explicitement la forme des cellules ci-dessus, la preuve du théorème principal (Théorème 2.3) étant élémentaire et purement combinatoire.

\section{Cellules de Kazhdan-Lusztig pour le groupe symétrique, correspondance de Robinson-Schensted}

Nous introduisons dans cette partie la relation d'équivalence $\sim_{L}$ permettant de définir les cellules de Kazhdan-Lusztig dans le cadre du groupe symétrique. Dans le cadre général des groupes de Coxeter, cette relation fait appel à la théorie de Kazhdan-Lusztig et en particulier à la base de Kazhdan-Lusztig associée à l'algèbre de Hecke du groupe de Coxeter (voir [KL]). Cependant, dans le cadre qui nous intéresse ici, Kazhdan et Lusztig ont montré que cette relation se définit

Classification mathématique par sujets (2000). - 20F55. 
complètement élémentairement à l'aide de la correspondance de Robinson-Schensted (voir par exemple $[\mathbf{A r}])$.

Soit $n \in \mathbb{N}$ et soit $\mathfrak{S}_{n}$ le groupe symétrique en $n$ éléments. Pour $i \in\{1, \ldots, n-1\}$, nous désignons par $s_{i}$ la transposition $(i i+1)$. Alors $\mathfrak{S}_{n}$ a une présentation par :

- générateurs : $S=\left\{s_{1}, s_{2}, \ldots, s_{n-1}\right\}$;

- relations :

$$
\begin{array}{ll}
s_{i}^{2}=1 & \text { pour } i=1, \ldots, n-1 \\
s_{i} s_{i+1} s_{i}=s_{i} s_{i+1} s_{i} & \text { pour } i=1, \ldots, n-2 \\
s_{j} s_{i}=s_{i} s_{j} & \text { pour }|i-j|>1 .
\end{array}
$$

Nous allons maintenant introduire la relation d'équivalence $\sim_{L}$. Soit $l: \mathfrak{S}_{n} \rightarrow \mathbb{N}$ la fonction longueur usuelle définie sur $\mathfrak{S}_{n}$. Soit $w \in \mathfrak{S}_{n}$, alors l'ensemble de descente (à gauche) de $w$ est l'ensemble suivant :

$$
L(w)=\{s \in S \mid l(s w)<l(w)\} .
$$

Soit maintenant $x$ et $y$ deux éléments de $\mathfrak{S}_{n}$ et soit $s \in S$, on écrit $x \sim_{L, s} w$ si et seulement si :

$-x=s w, l(s w)=l(w)+1$ et $L(w) \not \subset L(x)$,

- ou $w=s x, l(s x)=l(x)+1$ et $L(x) \not \subset L(w)$.

La relation d'équivalence $\sim_{L}$ est alors la clôture transitive de la relation $\sim_{L, s}$ c'est à dire que l'on a $x \sim_{L} y$ si et seulement si il existe des éléments $x_{0}=x, x_{1}, \ldots, x_{r-1}, x_{r}=y$ de $\mathfrak{S}_{n}$ et $s_{j_{0}}, s_{j_{1}}, \ldots, s_{j_{r-1}}$ des éléments de $S$ tels que $x_{i} \sim_{L, s_{j_{i}}} x_{i+1}$ pour $i=0, \ldots, r-1$. Les classes d'équivalence de $\sim_{L}$ sont appelées les cellules (à gauche) de Kazhdan-Lusztig. Elles permettent entre autres de construire les représentations irréductibles de l'algèbre de Hecke de type $A_{n-1}$.

Un critère agréable permet de vérifier si deux éléments de $\mathfrak{S}_{n}$ sont dans la même cellule : c'est la correspondance de Robinson-Schensted (voir $[\mathbf{F u}]$ pour un exposé détaillé de cette correspondance et de ses applications). Pour donner cette correspondance, introduisons quelques notations. Soit $\lambda=\left(\lambda_{1}, \ldots, \lambda_{r}\right)$ une partition de rang $n$ (tel que $\left.\lambda_{1} \geq \lambda_{2} \geq \ldots \geq \lambda_{r}\right)$. Le diagramme de Young de $\lambda$ est l'ensemble

$$
D(\lambda)=\left\{(i, j) \in \mathbb{N}_{>0} \times \mathbb{N}_{>0} \mid 1 \leq j \leq \lambda_{i}\right\} .
$$

Les éléments du diagramme de $\lambda$ sont appelés les boîtes de $\lambda$.

On associe maintenant à chaque élément $w \in \mathfrak{S}_{n}$ une paire de tableaux de Young standard $(P(w), Q(w))$ de même forme $\lambda=\left(\lambda_{1}, \ldots, \lambda_{r}\right)$, une partition de $n$ (c'est à dire que les tableaux $P(w)$ et $Q(w)$ de forme $\lambda$ sont remplies par les entiers $\{1, \ldots, n\}$ de telle sorte que les coefficients sont disposés en ordre croissant dans chaque ligne de gauche à droite et dans chaque colonne de haut en bas).

Exemple 1.1. - Ci-dessous un tableau standard de forme $\lambda=(4,2,2,1,1)$

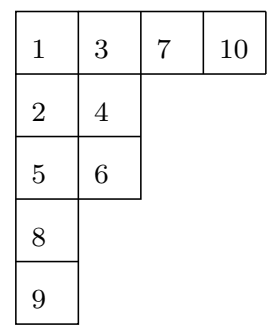

Au départ, $P(w)$ et $Q(w)$ sont vides. Pour tout $i=1, \ldots, n$, on insère récursivement sur les lignes de $P(w)$ l'entier $w(i)$ de façon à obtenir un tableau standard. Si tous les entiers situés sur la première ligne de $P(w)$ sont inférieurs à $w(i)$, on insère $w(i)$ sur la première ligne. Sinon, on remplace le plus petit entier $j$ supérieur à $w(i)$ par $w(i)$ et on insère $j$ sur la ligne suivante. Le processus débute sur la première ligne et s'arrête lorsque l'entier inséré ne prend la place d'aucun autre. Parallèlement, on note dans $Q(w)$ l'ordre d'apparition des boîtes. On obtient une application 
qui est en fait une bijection :

$$
\begin{aligned}
\mathfrak{S}_{n} & \rightarrow \bigcup_{\lambda \in \Pi_{n}} T_{\lambda} \times T_{\lambda} \\
w & \mapsto(P(w), Q(w))
\end{aligned}
$$

où $T_{\lambda}$ désigne l'ensemble des tableaux standard de forme $\lambda$ et $\Pi_{n}$ l'ensemble des partitions de $n$.

Exemple 1.2. - Ci-dessous la correspondance de Robinson-Schensted pour $\mathfrak{S}_{3}$.

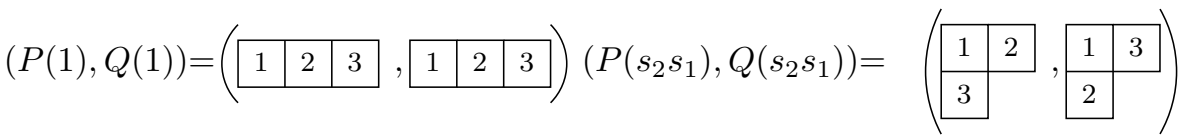

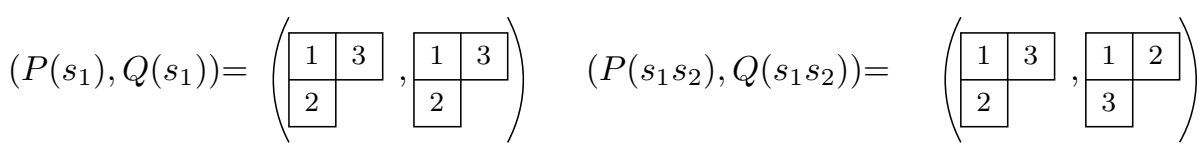

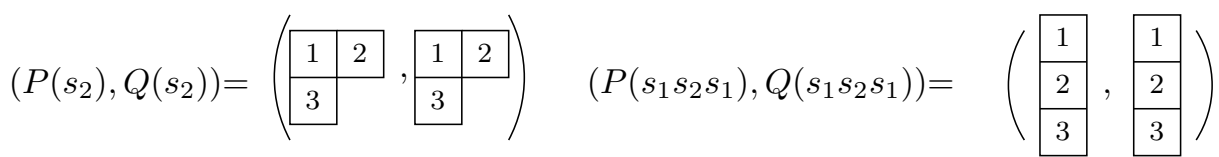

Finalement, deux éléments $w_{1}$ et $w_{2}$ sont dans la même cellule à gauche si et seulement si $Q\left(w_{1}\right)=Q\left(w_{2}\right)$. Ceci fournit donc un algorithme très efficace pour tester si deux éléments sont dans la même cellule.

\section{Calcul explicite}

Nous gardons les notations adoptées dans les sections précédentes auxquelles nous ajoutons les suivantes. Pour $i \leq j<n$, nous notons

$$
r_{(i)}^{(j)}:=s_{j} s_{j-1} \ldots s_{i}
$$

et

$$
w_{(i)}^{(j)}:=s_{i} s_{i+1} s_{i} \ldots s_{j} s_{j-1} \ldots s_{i}=r_{(i)}^{(i)} r_{(i)}^{(i+1)} \ldots r_{(i)}^{(j)} .
$$

Considérons une partition $\lambda=\left(\lambda_{1}, \lambda_{2}, \ldots, \lambda_{p}\right)$ de $n$ (où on suppose que $\lambda_{1} \geq \lambda_{2} \geq \ldots \geq \lambda_{p}$ ). Soit $\mathfrak{S}_{\lambda}$ le sous groupe parabolique de $\mathfrak{S}_{n}$ correspondant. Il est engendré par la partie $\left\{s_{i_{1}}, s_{i_{2}}, \ldots, s_{i_{k}}\right\}$ de $S$ où l'ensemble $\left\{i_{1}, i_{2}, \ldots, i_{k}\right\}$ est obtenu en enlevant les entiers $\lambda_{1}, \lambda_{1}+\lambda_{2}, \ldots, \sum_{j=1}^{p-1} \lambda_{j}$ de $\{1,2, \ldots, n\}$. Soit maintenant $w_{\lambda}$ l'élément de longueur maximale dans $\mathfrak{S}_{\lambda}$. Il est bien connu que :

$$
w_{\lambda}=w_{(1)}^{\left(\lambda_{1}-1\right)} w_{\left(\lambda_{1}+1\right)}^{\left(\lambda_{1}+\lambda_{2}-1\right)} \ldots w_{\left(\sum_{i=1}^{j} \lambda_{i}+1\right)}^{\left(\sum_{i=1}^{j+1} \lambda_{i}-1\right)} \ldots w_{\left(\sum_{i=1}^{p-1} \lambda_{i}+1\right)}^{(n-1)} .
$$

Notons $\Gamma_{\lambda}$ la cellule (à gauche) contenant l'élément $w_{\lambda}$. Le but de cette note est de déterminer explicitement cet ensemble $\Gamma_{\lambda}$. Par [Lu, 5.26.1] (résultat de Barbasch et Vogan, généralisé par Geck dans $[\mathbf{G e}])$, il existe une partie $X_{\lambda}$ de $\mathfrak{S}_{n}$ vérifiant :

$$
\Gamma_{\lambda}=\left\{x w_{\lambda} \mid x \in X_{\lambda}\right\},
$$

et tel que pour tout $x \in X_{\lambda}$, on a $l\left(x w_{\lambda}\right)=l(x)+l\left(w_{\lambda}\right)$.

Lemme 2.1. - Soit $\lambda=\left(\lambda_{1}, \lambda_{2}, \ldots, \lambda_{p}\right)$ une partition de $n$. Soit $j \in[1, p-1]$ tel que $\lambda_{j} \neq \lambda_{j+1}$. Alors :

$$
\underset{\left(\sum_{i=1}^{j} \lambda_{i}\right)}{r^{(n-1)}} \in X_{\lambda}
$$


Preuve. - On construit pour $w_{\lambda}$ et $r_{j}^{(n-1)} w_{\lambda}$ les tableaux de Young $Q\left(w_{\lambda}\right)$ et $Q\left(r^{(n-1)} w_{\lambda}\right)$

$$
\left.\left(\sum_{i=1}^{j} \lambda_{i}\right) \text { ( } \sum_{i=1}^{j} \lambda_{i}\right)
$$

donnés par la correspondance de Robinson-Schensted. On obtient dans les deux cas le même tableau. Ceci prouve que ces deux éléments sont dans la même cellule à gauche.

Nous avons maintenant le résultat suivant :

Lemme 2.2. - Soit $\lambda=\left(\lambda_{1}, \lambda_{2}, \ldots, \lambda_{p}\right)$ une partition de $n$, on suppose que $\lambda_{j} \neq \lambda_{j+1}$ pour $j \in[1, p-1]$, alors :

$$
r_{\left(\sum_{i=1}^{(n-1)} \lambda_{i}\right)} w_{\lambda}=w_{\left(\lambda_{1}, \ldots, \lambda_{j-1}, \lambda_{j}-1, \lambda_{j+1}, \ldots, \lambda_{p}\right)} r_{\left(\sum_{i=1}^{j-1} \lambda_{i}+1\right)}^{(n-1)}
$$

Preuve. - On a :

$$
w_{\lambda}=w_{(1)}^{\left(\lambda_{1}-1\right)} w_{\left(\lambda_{1}+1\right)}^{\left(\lambda_{1}+\lambda_{2}-1\right)} \ldots w_{\left(\sum_{i=1}^{j} \lambda_{i}+1\right)}^{\left(\sum_{i=1}^{j+1} \lambda_{i}-1\right)} \ldots w_{\left(\sum_{i=1}^{p-1} \lambda_{i}+1\right)}^{(n-1)}
$$

On obtient donc :

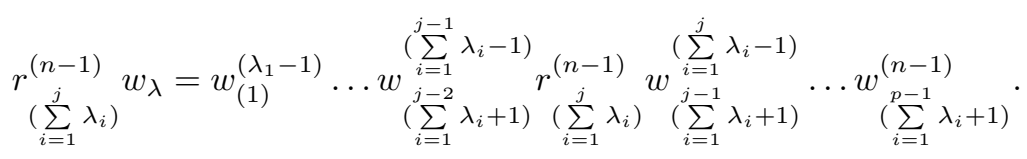

En utilisant les relations de $\mathfrak{S}_{n}$, on obtient :

De plus, on a :

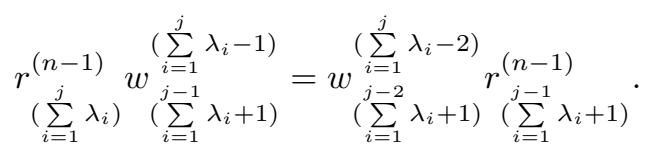

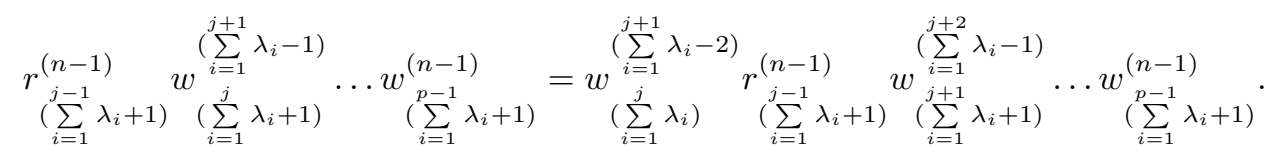

On en déduit, par récurrence :

ce qui conclut la preuve.

$$
r_{\left(\sum_{i=1}^{(n-1)} \lambda_{i}\right)}^{(n)} w_{\lambda}=w_{\left(\lambda_{1}, \ldots, \lambda_{j-1}, \lambda_{j}-1, \lambda_{j+1}, \ldots, \lambda_{p}\right)} \underset{\left(\sum_{i=1}^{(n-1} \lambda_{i}+1\right)}{(n-1)}
$$

Nous pouvons maintenant énoncer le théorème principal :

Théoreme 2.3. - Soit $\lambda=\left(\lambda_{1}, \lambda_{2}, \ldots, \lambda_{p}\right)$ une partition de $n$. En utilisant les notations introduites ci-dessus, on a:

$$
X_{\lambda}=\bigcup_{j=1}^{p} X_{\left(\lambda_{1}, \lambda_{2}, \ldots, \lambda_{j}-1, \ldots, \lambda_{p}\right)} r_{\left(\sum_{i=1}^{(n-1)} \lambda_{i}\right)}^{(j}
$$

en prenant comme convention $: r_{(i)}^{(j)}=1$ si $j<i$ et $X_{\left(\mu_{1}, \mu_{2}, \ldots, \mu_{p}\right)}=\{0\}$ si $\left(\mu_{1}, \mu_{2}, \ldots, \mu_{p}\right)$ n'est pas une partition.

Preuve. - On commence par montrer que si $\left(\lambda_{1}, \ldots, \lambda_{j}-1, \ldots, \lambda_{p}\right)$ est une partition de $n-1$, on a :

$$
X_{\left(\lambda_{1}, \ldots, \lambda_{j}-1, \ldots, \lambda_{p}\right)} r_{\left(\sum_{i=1}^{(n-1)} \lambda_{i}\right)}^{j} \subset X_{\left(\lambda_{1}, \ldots, \lambda_{j}, \ldots, \lambda_{p}\right)} .
$$

Soit donc $x \in X_{\left(\lambda_{1}, \ldots, \lambda_{j}-1, \ldots, \lambda_{p}\right)}$, d'après le lemme 2.2 , on a :

$$
r_{\left(\sum_{i=1}^{(n-1)} \lambda_{i}\right)}^{(n)} w_{\lambda}=w_{\left(\lambda_{1}, \ldots, \lambda_{j-1}, \lambda_{j}-1, \lambda_{j+1}, \ldots, \lambda_{p}\right)} r_{\left(\sum_{i=1}^{(n-1} \lambda_{i}+1\right)}^{(n-1)}
$$


Il suit donc :

$$
x r_{\left(\sum_{i=1}^{(n-1)} \lambda_{i}\right)}^{j} w_{\lambda}=x w_{\left(\lambda_{1}, \ldots, \lambda_{j-1}, \lambda_{j}-1, \lambda_{j+1}, \ldots, \lambda_{p}\right)} r_{\left(\sum_{i=1}^{(n-1} \lambda_{i}+1\right)}^{(n-1)} .
$$

Par définition, il existe une suite $\left(s_{i_{l}}\right)_{l=1, \ldots, m}$ d'éléments de $\left\{s_{1}, \ldots, s_{n-2}\right\}$ (le système de générateurs de $\left.\mathfrak{S}_{n-1}\right)$ telle que :

$$
w_{\left(\lambda_{1}, \ldots, \lambda_{j-1}, \lambda_{j}-1, \lambda_{j+1}, \ldots, \lambda_{p}\right)} \sim_{L, s_{i_{1}}} \ldots \sim_{L, s_{i_{m}}} x w_{\left(\lambda_{1}, \ldots, \lambda_{j-1}, \lambda_{j}-1, \lambda_{j+1}, \ldots, \lambda_{p}\right)}
$$

On peut composer par $r_{\left(\sum_{i=1}^{(n-1)} \lambda_{i}+1\right)}^{j-1}$ à droite car les éléments $s_{i_{l}}$ intervenant dans les équivalences sont dans $\mathfrak{S}_{n-1}$, on obtient donc :

$$
\begin{aligned}
w_{\left(\lambda_{1}, \ldots, \lambda_{j-1}, \lambda_{j}-1, \lambda_{j+1}, \ldots, \lambda_{p}\right)} r_{\left(\sum_{i=1}^{(n-1} \lambda_{i}+1\right)}^{(n-1)} & \sim_{L, s_{i 1}} \ldots \\
\ldots & \sim_{L, s_{i m}} x w_{\left(\lambda_{1}, \ldots, \lambda_{j-1}, \lambda_{j}-1, \lambda_{j+1}, \ldots, \lambda_{p}\right)} r_{\left(\sum_{i=1}^{j-1} \lambda_{i}+1\right)}^{(n-1)}
\end{aligned}
$$

En utilisant le lemme 2.2, il suit :

$$
r_{\left(\sum_{i=1}^{(n-1)} \lambda_{i}\right)}^{\left(n-\lambda_{1}, \ldots, \lambda_{p}\right)} \sim_{L, s_{i_{1}}} \ldots \sim_{L, s_{i}} \operatorname{xr}_{\left(\sum_{i=1}^{(n-1)} \lambda_{i}\right)}^{(n-1)} w_{\left(\lambda_{1}, \ldots, \lambda_{p}\right)} .
$$

Or, d'après le lemme 2.1, on a :

$$
r_{\left(\sum_{i=1}^{(n-1)} \lambda_{i}\right)}^{j} \in X_{\lambda}
$$

il suit donc :

$$
w_{\left(\lambda_{1}, \ldots, \lambda_{p}\right)} \sim_{L} r_{\left(\sum_{i=1}^{(n-1)} \lambda_{i}\right)}^{(n-1)} w_{\left(\lambda_{1}, \ldots, \lambda_{p}\right)}
$$

d'où :

$$
w_{\left(\lambda_{1}, \ldots, \lambda_{p}\right)} \sim_{L} x r_{\left(\sum_{i=1}^{(n-1)} \lambda_{i}\right)}^{j} w_{\left(\lambda_{1}, \ldots, \lambda_{p}\right)}
$$

On a donc montré :

$$
\bigcup_{j=1}^{p} X_{\left(\lambda_{1}, \lambda_{2}, \ldots, \lambda_{j}-1, \ldots, \lambda_{p}\right)} r_{\left(\sum_{i=1}^{(n-1)} \lambda_{i}\right)}^{(n-1)} \subset X_{\left(\lambda_{1}, \lambda_{2}, \ldots, \lambda_{p}\right)} .
$$

Notons également que les éléments de $\bigcup_{j=1}^{p} X_{\left(\lambda_{1}, \lambda_{2}, \ldots, \lambda_{j}-1, \ldots, \lambda_{p}\right)} r_{\left(\sum_{i=1}^{(n-1)} \lambda_{i}\right)}^{(n)}$ sont tous distincts. On conclut la preuve par cardinalité : le cardinal des cellules $\Gamma_{\lambda}$ correspond aux dimensions des représentations irréductibles de $\mathfrak{S}_{n}$. On a :

$$
\left|\Gamma_{\lambda}\right|=\left|X_{\lambda}\right|
$$

D'après [Fu, 4.3.8], on a :

$$
\left|X_{\lambda}\right|=\sum_{j=1}^{p}\left|X_{\left(\lambda_{1}, \lambda_{2}, \ldots, \lambda_{j}-1, \ldots, \lambda_{p}\right)}\right|
$$

avec $\left|X_{\left(\lambda_{1}, \lambda_{2}, \ldots, \lambda_{j}-1, \ldots, \lambda_{p}\right)}\right|=0$ si $\left(\lambda_{1}, \lambda_{2}, \ldots, \lambda_{j}-1, \ldots, \lambda_{p}\right)$ n'est pas une partition. Il suit donc :

$$
\bigcup_{j=1}^{p} X_{\left(\lambda_{1}, \lambda_{2}, \ldots, \lambda_{j}-1, \ldots, \lambda_{p}\right)} r_{\left(\sum_{i=1}^{(n-1)} \lambda_{i}\right)}^{(n-1)}=X_{\left(\lambda_{1}, \lambda_{2}, \ldots, \lambda_{p}\right)}
$$

ce qu'il fallait montrer.

Le corollaire suivant va maintenant nous donner la forme explicite des éléments composant la cellule $\Gamma_{\lambda}$. Notons que la donnée de ce type de cellules suffit pour construire toutes les représentations irréductibles de $\mathfrak{S}_{n}$. Introduisons quelques notations supplémentaires. Soit $\lambda$ une partition de $n$ avec $n_{1}(\lambda)$ parts égales à $1, n_{2}(\lambda)$ parts égale à $2, \ldots, n_{r}(\lambda)$ égales à $r$. On notera alors $\lambda=$ $\left(1^{n_{1}(\lambda)}, \ldots,(i-1)^{n_{i-1}(\lambda)}, i^{n_{i}(\lambda)}, \ldots, r^{n_{r}(\lambda)}\right)$. De plus, si $n_{k}(\lambda) \neq 0$, on notera $\lambda^{(i)}$ la partition 
$\left(1^{n_{1}(\lambda)}, \ldots,(i-1)^{n_{i-1}(\lambda)+1}, i^{n_{i}(\lambda)-1}, \ldots, r^{n_{r}(\lambda)}\right)$ de $n-1$. Le résultat suivant est une conséquence direct du Théorème 2.3.

Corollaire 2.4. - Soit $\lambda=\left(1^{n_{1}(\lambda)}, \ldots,(i-1)^{n_{i-1}(\lambda)}, i^{n_{i}(\lambda)}, \ldots, r^{n_{r}(\lambda)}\right)$ une partition de $n$. Soit $X_{\lambda}$ comme dans le Théorème 2.3. Alors, $x \in X_{\lambda}$ si et seulement si, il existe une suite d'entiers $i_{j} \in[1, r]$ avec $j=2, \ldots, n$ vérifiant $n_{i_{j}}\left(\lambda^{\left(i_{j+1}, \ldots, i_{n}\right)}\right) \neq 0$ et :

$$
\begin{array}{r}
x=r_{\left(\sum_{k=i_{2}}^{r} k n_{k}\left(\lambda^{\left(i_{3}, i_{4}, \ldots, i_{n}\right)}\right)\right)}^{(1)} r_{\left(\sum_{k=i_{3}}^{r} k n_{k}\left(\lambda^{\left(i_{4}, i_{5}, \ldots, i_{n}\right)}\right)\right)}^{(2)} \cdots \\
\ldots r_{\left(\sum_{k=i_{n-1}}^{r} k n_{k}\left(\lambda^{\left(i_{n}\right)}\right)\right)}^{(n-2)} r_{\left(\sum_{k=i_{n}}^{r} k n_{k}(\lambda)\right)}^{(n-1)} .
\end{array}
$$

$y \in \Gamma_{\lambda}$ si et seulement si il existe une suite d'entiers $i_{j} \in[1, r]$ avec $j=2, \ldots, n$ vérifiant :

$$
\begin{array}{r}
y=r_{\left(\sum_{k=i_{2}}^{r} k n_{k}\left(\lambda^{\left(i_{3}, i_{4}, \ldots, i_{n}\right)}\right)-i_{2}+1\right)}^{(1)} r_{\left(\sum_{k=i_{3}}^{r} k n_{k}\left(\lambda^{\left(i_{4}, i_{5}, \ldots, i_{n}\right)}\right)-i_{3}+1\right)}^{(2)} \cdots \\
\ldots r_{\left(\sum_{k=i_{n-1}}^{r} k n_{k}\left(\lambda^{\left(i_{n}\right)}\right)-i_{n-1}+1\right)}^{(n-2)} r_{\left(\sum_{k=i_{n}}^{r} k n_{k}(\lambda)-i_{1}+1\right)}^{(n-1)} .
\end{array}
$$

\section{Références}

[Ar] S. Ariki, Robinson-Schensted correspondence and left cells, Comb. meth. in rep. theory (Kyoto, 1998), Adv. Studies in Pure Math., 28, 2000.

[Fu] W. Fulton, Young tableaux, London Math. Soc. Student Texts, 35, Cambridge University Press, Cambridge, 1997.

[Ge] M. Geck, On the induction of Kazhdan-Lusztig cells, Bull. London. Math. Soc., 608-614, 35, 2003.

[KL] D. Kazhdan, G. Lusztig, Representations of Coxeter groups and Hecke algebras, Invent. Math. 53, 1979.

[Lu] G. Lusztig, Left cells in Weyl groups. Lie group representations, I, 99-111, Lecture Notes in Math., 1024, Springer, Berlin, 1983.

[Lu2] G. Lusztig, Intersection chomology methods in representation theory, Proceedings of the International Congress of Mathematics, Kyoto, Japan, (I. Satake, ed.), Springer-Verlag, 1991, 155-174, 1990.

9 novembre 2006

NiCOLAS JACON, UFR Sciences et techniques - 16, route de Gray - 25030 Besançon cedex.

E-mail : jacon@math.univ-fcomte.fr 